\title{
Cyclodextrin-based nanosponges as vehicles for antiviral drugs: challenges and perspectives
}

\author{
David Lembo1, Francesco Trotta² \& Roberta Cavalli*,3 \\ ${ }^{1}$ Department of Clinical \& Biological Sciences, University of Turin, S. Luigi Gonzaga Hospital, Regione Gonzole, 10, 10043 \\ Orbassano, Torino, Italy \\ ${ }^{2}$ Department of Chemistry, University of Turin, Via P. Giuria 7, 10125, Torino, Italy \\ ${ }^{3}$ Department of Drug Science \& Technology, University of Turin, Via P. Giuria 9, 10125, Torino, Italy \\ * Author for correspondence: Tel.: +39 011670 7190; Fax: +390116707687; roberta.cavalli@unito.it
}

\section{"All together, the features outlined make $\beta C D$-NS a promising platform for the development of innovative and advanced antiviral therapeutic treatments to fight viral infections."}

\section{Keywords:antiviral drugs $\bullet \beta$-cyclodextrins $\bullet$ nanosponges $\bullet$ viral infections}

Viral diseases affect billions of people each year worldwide causing million of deaths. Despite the accumulation of a large body of knowledge about the replicative and pathogenetic mechanisms of many human viral pathogens, approved antiviral drugs are available only for a restricted array of viruses. These include the HIV, the HSV, the varicella zoster virus, the human CMV, the influenza virus and the HBV and HCV [1]. This scenario is further complicated by pharmaceutical/pharmacological issues that limit the therapeutic potential of the current available drugs.

A promising strategy to overcome these limitations is the development of nanoscale carriers for the delivery of antiviral drugs, in other words, nanomedicine formulations.

Their use can offer specific advantages that can improve antiviral treatments, considering the narrow therapeutic indices, high doses and frequent administrations needed for a number of antivirals drugs due to their limited aqueous solubility, low permeability, incomplete biodistribution and short half-life $[2,3]$.

Indeed, nanocarriers modify the physicochemical properties of the incorporated molecules, affect the pharmacokinetics profiles and allow to overcome biological barriers, thereby allowing to enhance the effectiveness of the drug and decrease severe side effects [4].

In addition, drug-targeting nanomedicine strategies based on the recognition of viral molecules expressed on the surface of infected cells might facilitate the site-specific release of antiviral drugs, thus permitting to decrease the administered dose regimen of antivirals.

To date, a number of nanocarriers, either inorganic or organic, have been developed to improve the delivery and therapeutic efficacy of antiviral medicines [2,3].

Among the various nanomaterials that have been designed as potential nanocarriers, cyclodextrin-based polymers are of particular interest for a number of reasons highlighted by this article.

Cyclodextrins are a class of cyclic glucopyranose oligomers, obtained from starch by enzymatic action, with a characteristic toroidal shape that forms a truncated cone-shaped lipophilic cavity. The main common native cyclodextrins are named $\alpha, \beta$ and $\gamma$ which comprise six, seven and eight glucopyranose units, respectively. Cyclodextrins have the capability to include compounds whose size and polarity are compatible with that of their cavity [5].

Indeed, some studies have shown that $\beta$-cyclodextrins are able to include antiviral drugs forming inclusion complexes and enhancing their efficacy $[6,7]$.

A further development in the field is represented by $\beta$-cyclodextrin-based nanosponges $(\beta C D-N S)$ designed to magnify drug complexation capability. They are solid nanoparticles with nanocavities, consisting of highly cross-linked cyclodextrin units $[8,9]$. 
The most relevant advantages of $\beta C D-N S$ are their cost effective and versatile preparation method. This supramolecular material is prepared by reacting cyclodextrins with various cross-linker agents, generally an activated carbonyl compound, such as carbonyldiimidazole, dianhydride or carboxylic acids. The reaction can be optimized to produce nanoparticles with specific chemical structure, sizes and surface charges. Moreover, stimuli-responsive nanosponges with a precise capacity to control drug release in response of external environmental changes have been designed $[10,11]$. For instance, redox $/ \mathrm{pH}$-sensitive $\beta \mathrm{CD}$-NS have been recently developed for the targeted intracellular release of anticancer drugs [12]. In addition, it is possible to exploit active targeting strategies, in other words, the conjugation of receptor ligands on nanosponge surface, to promote the drug site-specific release.

Another interesting feature of $\beta C D-N S$ is the marked capability to entrap molecules with different structures as well as macromolecules and gases. The cooperation of various cyclodextrin cavities and the polymer network present in the nanostructure can play an important role for the drug loading. Indeed, the drugs can be incorporated within nanosponges either as inclusion complexes or as noninclusion complexes, exploiting the various interaction sites available belonging to the cyclodextrin cavities and the polymer meshes. It is worth noting that the molecular loading allows the increase of the apparent solubility and the dissolution rate of drugs due to the loss of crystallinity.

Another important characteristic of the loading within the $\beta C D-N S$ is the achievement of prolonged and constant drug release kinetics, easily tunable with the chemical structure of the system. Particularly, the loaded drug is released in a slow and controlled manner due to the drug diffusion mechanism through the polymer network, following pseudo zero-order kinetics.

Of note, the incorporation can also protect the drug from degradation and minimize its systemic exposure.

As for their dosage forms and routes of administration, nanosponges can be generally formulated as stable aqueous nanosuspensions or solid dosage forms (i.e., capsules) and can be administered by various routes including parenteral, oral and topical.

Concerning safety, $\beta C D-N S$ are biocompatible, biodegradable and show negligible toxicity in preclinical studies. The acute systemic toxicity of blank (unloaded) $\beta C D-N S$ was evaluated in mice following the administration of doses upto $5000 \mathrm{mg} / \mathrm{kg}$. Mice showed no toxicity signs or adverse reactions [13]. The oral administration of the $\beta C D-N S$ was also investigated both in mice and rats with no apparent side effects [13].

More recent studies showed that drug-loaded $\beta C D-N S$, administered either intravenously or orally, have been able to enhance the drug effectiveness in animal models [8].

Interestingly, $\beta C D-N S$ can promote drug absorption from the GI tract, following oral administration. Torne et al. showed that the oral bioavailability of paclitaxel was increased about threefold after the oral administration of paclitaxel-loaded $\beta C D-N S$ to rats, in comparison to the control (commercially available TAXOL ${ }^{\circledR}$, Bristol-Myers Squibb Company, Chester, UK) [14].

Altogether, the features discussed above could be advantageously translated into the fields of antivirals, making $\beta C D-N S$ a suitable nanoplatform for the delivery of antiviral drugs. $\beta C D-N S$ as antiviral carriers were investigated, particularly to address poor water solubility problems. Indeed, the dissolution rate and the solubility of solid antiviral drugs represent limiting factors for their oral bioavailability. This is of particular interest for anti-HIV drugs, with the majority of them being orally administered.

For instance, nelfinavir is a protease inhibitor with low bioavailability used to treat HIV infections. To overcome this limitation, Vavia and coworkers prepared nelfinavir mesylate-loaded nanosponges to enhance the solubility of the drug [15].

More recently, efavirenz, a non-nucleoside reverse transcriptase inhibitor with a low aqueous solubility, was loaded in $\beta C D$-NS with the same aim. For this purpose, a ternary system, comprising drug-loaded $\beta C D-N S$ and polyvinylpyrrolidone $30 \mathrm{~K}$ was prepared. This ternary system produced a twofold increase in the bioavailability of efavirenz in comparison to the free drug after oral administration to rats [16].

Zainuddin and coworkers [17] developed $\beta C D$-NS using a microwave-mediated method, for improving the oral bioavailability of rilpivirine, a non-nucleoside inhibitor of HIV reverse transcriptase. A significant increase of the drug pharmacokinetics parameters (i.e., $\mathrm{C}_{\max }$ and $\mathrm{AUC}$ values) was obtained after the oral administration of rilpivirine loaded in $\beta \mathrm{CD}-\mathrm{NS}$ to rats, in comparison to the plain drug.

$\beta C D-N S$ were also exploited for enhancing the solubility and bioavailability of acyclovir, a drug widely used for the therapy of HSV infections.

Purposely-tuned carboxylated $\beta \mathrm{CD}$-NS, containing dissociable carboxylic groups in their structure, were developed for its encapsulation. The synthesis rationale was to obtain further electrostatic contributions for drug complexation, in addition to the cyclodextrin cavities. Electrostatic interactions may occur between the carboxylic 
groups present in the nanosponge structure and the amino group of acyclovir. Acyclovir-loaded carboxylated $\beta C D-$ NS [18] showed a high drug loading suitable for the solubility enhancement and prolonged in vitro release kinetics with a $20 \%$ drug release after $3 \mathrm{~h}$.

Nanosponge nanotechnology might provide other valuable applications for the improvement of antivirals.

They are a suitable vehicle for multiple drugs at the same time. This makes $\beta \mathrm{CD}-\mathrm{NS}$ an attractive formulation strategy for antiviral therapies that rely on drug combination, such as the highly active antiretroviral therapy currently used to treat HIV-infected individuals.

In addition, $\beta C D-N S$ might represent a new avenue for controlling the amount, the dosage frequency and the delivery site of antivirals.

In this context, the structure of $\beta C D$-NS might be further tuned for the site-specific drug release potential. They could be engineered, modifying their surfaces to target infected cells and release within cell their cargo as a 'Trojan horse', or to compete with cell-surface receptors used by viruses for cell attachment by mimicking them. This strategy may be useful either for therapy or prevention of viral diseases.

The local and specific release of antiviral drugs can decrease the administered doses and minimize damage to healthy cells.

Besides small molecules, the use of silencing RNAs is an alternative antiviral strategy [19]. However, this approach requires the development of specific therapeutic formulations. The nanostructure of $\beta C D-N S$ might be optimized for storing and delivering antiviral silencing RNAs, considering their capability to protect sensitive molecules like peptides or proteins.

All together, the features outlined make $\beta C D-N S$ a promising platform for the development of innovative and advanced antiviral therapeutic treatments to fight viral infections. However, several points remain to be addressed to optimize $\beta C D-N S$ as antiviral vehicles. These include upscaled production, long-term stability, improvement of drug payload and deeper evaluation of the toxicity.

\section{Financial \& competing interests disclosure}

The authors have no relevant affiliations or financial involvement with any organization or entity with a financial interest in or financial conflict with the subject matter or materials discussed in the manuscript. This includes employment, consultancies, honoraria, stock ownership or options, expert testimony, grants or patents received or pending, or royalties.

No writing assistance was utilized in the production of this manuscript.

\section{References}

1 De Clercq E, Li G. Approved antiviral drugs over the past 50 years. Clin. Microbiol. Rev. 29(3), 695-747 (2016).

2 Lembo D, Donalisio M, Civra A, Argenziano M, Cavalli R. Nanomedicine formulations for the delivery of antiviral drugs: a promising solution for the treatment of viral infections. Expert Opin. Drug Deliv. 15(1), 93-114 (2018).

3 Lembo D, Cavalli R. Nanoparticulate delivery systems for antiviral drugs. Antivir. Chem. Chemother. 21, 53-70 (2010).

4 Prasad M, Lambe UP, Brar B et al. Nanotherapeutics: an insight into healthcare and multidimensional applications in medical sector of the modern world. Biomed. Pharmacother. 97, 1521-1537 (2018).

5 Jansook P, Ogawa N, Loftsson T. Cyclodextrins: structure, physicochemical properties and pharmaceutical applications. Int. J. Pharm. 535(1-2), 272-284 (2017).

6 Nair AB, Attimarad M, Al-Dhubiab BE, Wadhwa J, Harsha S, Ahmed M. Enhanced oral bioavailability of acyclovir by inclusion complex using hydroxypropyl- $\beta$-cyclodextrin. Drug Deliv. 21(7), 540-547 (2014).

7 Gilsoni RJ, Castro EF, Cavallaro LV, Moglioni AG, Sosnik A. Complexation of a 1-indanone thiosemicarbazone with hydroxypropyl- $\beta$-cyclodextrin enhances its activity against a hepatitis $C$ virus surrogate model. J. Nanosci. Nanotech. 15(6), 4224-4228 (2015).

8 Trotta F, Dianzani C, Caldera F, Mognetti B, Cavalli R. The application of nanosponges to cancer drug delivery. Expert Opin. Drug Deliv. 11(6), 931-941 (2014).

9 Swaminathan S, Cavalli R, Trotta F. Cyclodextrin-based nanosponges: a versatile platform for cancer nanotherapeutics development. Wiley Interdiscip. Rev. Nanomed. Nanobiotechnol. 8(4), 579-601 (2016).

10 Caldera F, Tannous M, Cavalli R, Zanetti M, Trotta F. Evolution of cyclodextrin nanosponges. Int. J. Pharm. 531(2), 470-479 (2017).

11 Trotta F, Caldera F, Dianzani C, Argenziano M, Barrera G, Cavalli R. Glutathione bioresponsive cyclodextrin nanosponges. ChemPlusChem 81(5), 439-443 (2016).

12 Daga M, Ullio C, Argenziano M et al. GSH-targeted nanosponges increase doxorubicin-induced toxicity 'in vitro' and 'in vivo' in cancer cells with high antioxidant defenses. Free Radic. Biol. Med. 97, 24-37 (2016). 
13 Shende P, Kulkarni YA, Gaud RS et al. Acute and repeated dose toxicity studies of different $\beta$-cyclodextrin-based nanosponge formulations. J. Pharm. Sci. 104(5), 1856-1863 (2015).

14 Torne SJ, Ansari KA, Vavia PR, Trotta F, Cavalli R. Enhanced oral paclitaxel bioavailability after administration of paclitaxel-loaded nanosponges. Drug Deliv. 17(6), 419-425 (2010).

15 Trotta F, Zanetti M, Cavalli R. Cyclodextrin-based nanosponges as drug carriers. Beilstein. J. Org. Chem. 8, 2091 (2012).

16 Rao MR, Shirsath C. Enhancement of bioavailability of non-nucleoside reverse transciptase inhibitor using nanosponges. AAPS PharmSciTech 18(5), 1728-1738 (2017).

17 Zainuddin R, Zaheer Z, Sangshetti JN, Momin M. Enhancement of oral bioavailability of anti-HIV drug rilpivirine $\mathrm{HCl}$ through nanosponge formulation. Drug Dev. Ind. Pharm. 43(12), 2076-2084 (2017).

18 Lembo D, Swaminathan S, Donalisio M et al. Encapsulation of acyclovir in new carboxylated cyclodextrin-based nanosponges improves the agent's antiviral efficacy. Int. J. Pharm. 443(1), 262-272 (2013).

19 Saravolac EG, Wong JP, Cairns MJ. Recent patents in antiviral siRNAs. Recent Pat. Antiinfect. Drug Discov. 5(1), 44-57 (2010). 